\title{
Comparing the Sensitivity of Quantity Based and Useful Lifetime Based Fixed Lifetime Inventory Models to Changes in Costs
}

\author{
Izevbizua $\mathbf{O}$ and Apanapudor $\mathbf{J}$ \\ Department of Mathematics, University of Benin, Benin City/Delta state University Abraka \\ Correspondence Author: Izevbizua O, Department of Mathematics, University of Benin, Benin City/Delta state University Abraka. \\ E-mail:- maorobo@yahoo.com
}

Received date: 15 June 2019, Accepted date: 26 August 2019, Online date: 29 August 2019

Copyright: (C) 2019 Izevbizua O et al, This is an open-access article distributed under the terms of the Creative Commons Attribution License, which permits unrestricted use, distribution, and reproduction in any medium, provided the original author and source are credited.

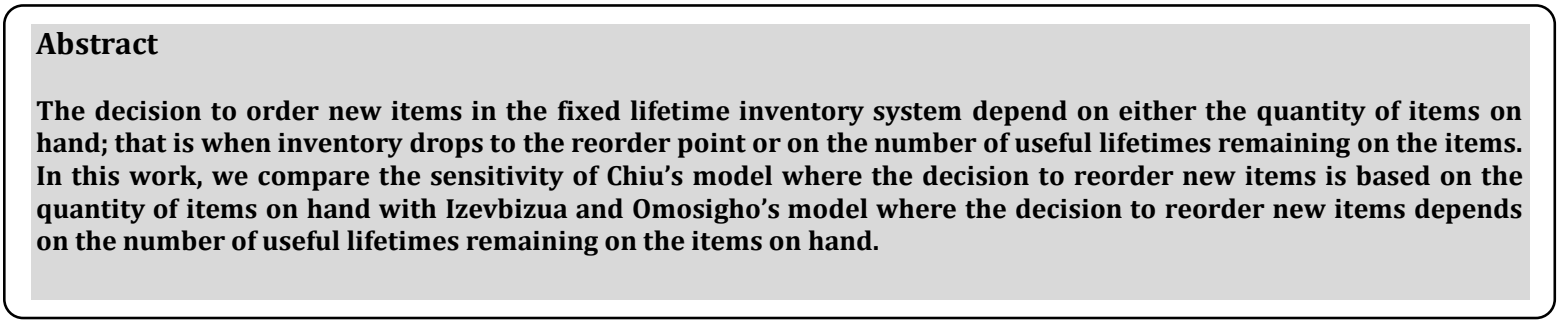

Keywords: fixed lifetime, quantity-based model, useful lifetime-based model, reorder, ordering policy

\section{INTRODUCTION}

The decision to reorder new items in the fixed lifetime inventory system depends on the number of items on hand or the number of the useful period(s) remaining on the items on hand. Authors in the literature with ordering policies based on the quantity of products on hand include; Chiu (1994), Bookbinder and Cakanyildirim (1999), Mohammad et al (2007), Hariga (2010) and Siriruk (2012) all of whom considered the ordering policy $(Q, r)$ which order $Q$ whenever inventory on hand drops to the reorder point $r$. Nahmias (1978), Hollier et al. (1995), Liu and Lian (1999) and Silver et al. (2012) considered the ordering policy $(s, S)$ which orders up to $S$ when inventory on hand drops to the reorder point $s$. Schmidt and Nahmias (1985), Perry and Posner (1998) and Olsson and Tydesjo (2010) considered the ordering policy $(S-1, S)$ in which an order is placed for exactly one item each time inventory is depleted by either demand or outdating. Shen et al. (2012) considered an ordering policy that maintains a minimum volume of inventory, whenever inventory drops to this level a new order is placed. As a deviation from these ordering policies, Izevbizua and Omosigho (2016) developed a new fixed lifetime inventory model where the decision to reorder is based on the number of useful life remaining on the items on hand rather than just the quantity of items on hand.

The policy takes the form $(y, m-1)$ interpreted as order ${ }^{y}$ when the useful lifetime remaining on the items on hand is one period. One advantage of this policy over existing policies is that the policy is not fixed. If the demand is high, the inventory manager can decide to reorder new items with two periods remaining on the items on hand instead of one period. This is very common during festive periods when sales are high. If the demand drops the inventory manager can reverse back to placing new orders with one useful period remaining on the items on hand. If new orders are placed with two periods remaining, the ordering

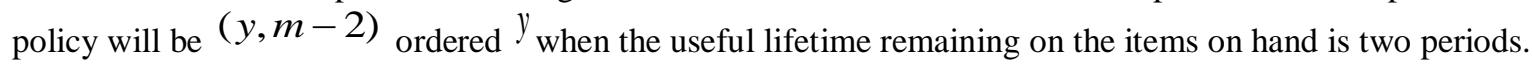

In this work, we implement Chiu's model (1994), obtain the ordering policy and compare the sensitivity of the ordering policy with the ordering policy obtained from the useful lifetime model developed by Izevbizua and Omosigho (2016). First we present Chiu's model and thereafter derive the total cost function for the useful lifetime model.

The total cost function obtained by Chiu (1994) is given. 
$E A C(Q, r)=\{(k+c Q+p E S+w E R) / E T\}+h O H$.

where

$E S=\int_{r}^{\infty}\left(d_{L}-r\right) f_{L}\left(d_{L}\right) d_{L}$

$E R=\int_{0}^{r+Q}\left(r+Q-d_{m+L}\right) f_{m+L}\left(d_{m+L}\right) d_{m+L}-\int_{0}^{r}\left(r-d_{m+L}\right) f_{m+L}\left(d_{m+L}\right) d_{m+L}$

$E T=\frac{Q-E R}{D}$

$O H=r-(D L)+\frac{Q}{2}$

so that

$E A C(Q, r)=\left\{k+c Q+p \int_{r}^{\infty}\left(d_{L}-r\right) f_{L}\left(d_{L}\right) d_{L}+w\left\{\int_{0}^{r+Q}\left(r+Q-d_{m+L}\right) f_{m+L}\left(d_{m+L}\right)-\right.\right.$

$$
\left.\left.\int_{0}^{r}\left(r-d_{m+L}\right) f_{m+L}\left(d_{m+L}\right) d_{m+L}\right\}\right\} / E T+h\left(r-D L+\frac{Q}{2}\right)
$$

where

$E S=$ shortage quantity

$E R=$ outdate quantity

$E T=$ cycle time

$\mathrm{OH}=$ on hand inventory

$Q=$ ordered quantity

$r=$ reorder point

$k=$ fixed ordering $\cos t$ per order

$c=$ replenishment $\cos t$ per unit

$p=$ shortage cost

$h=$ holding $\cos t$ per unit per unit time

$w=$ outdate $\cos t$ per unit

To implement the model, we obtain the partial derivative of equation (1) concerning the order quantity using Wolfram Mathematica 8 , equate to zero and solve the resulting equation.

$$
\begin{aligned}
& \frac{\partial E A C}{\partial Q}=\frac{h}{2}+\frac{D\left(c+\frac{1}{2}(Q+r)^{2} w f_{m+l}\right)}{Q+(\inf \text { inity })^{3} \frac{f_{l}}{3}-\frac{1}{2}(\inf \text { inity })^{2} r f_{l}+r^{3} \frac{f_{l}}{6}+\frac{r^{3} f_{m+l}}{6}-\frac{(Q+r)^{3}}{6} f_{m+l}}- \\
& \frac{D\left(1-\frac{1}{2}(Q+r)^{2} f_{m+l}\right)\left(k+c Q+p\left(\frac{\text { (inf } \text { inity })^{3} f_{l}}{3}-\frac{(\inf \text { inity })^{2} r f_{l}}{2}+\frac{r^{3} f_{l}}{6}\right)+w\left(-\frac{r^{3} f_{m+l}}{6}+\frac{\left.(Q+r)^{3} f_{m+l}\right)}{6}\right)\right.}{\left(Q+\frac{(\inf \text { inity })^{3} f_{l}}{3}-\frac{(\inf \text { inity })^{2} r f_{l}}{2}+\frac{r^{3} f_{l}}{6}+\frac{r^{3} f_{m+l}}{6}-\frac{(Q+r)^{3} f_{m+l}}{6}\right)^{2}}
\end{aligned}
$$
zero.

Going forward, we take $f$ (inf inity) to be zero and obtain an expression for the ordered quantity when the reorder point is

$$
\begin{aligned}
& \frac{h}{2}+\frac{D\left(c+\frac{Q^{2}}{2} w f_{m+l}\right)}{Q-\frac{Q^{3}}{6} f_{m+l}}-\frac{D\left(1-\frac{Q^{2}}{2} f_{m+l}\right)\left(k+c Q+w \frac{Q^{3}}{6} f_{m+l}\right)}{\left(Q-\frac{Q^{3}}{6} f_{m+l}\right)^{2}}=0 \\
& h\left(Q-\frac{Q^{3} f_{m+l}}{6}\right)^{2}+2 D\left(c+\frac{Q^{2} w f_{m+l}}{2}\right)\left(Q-\frac{Q^{3} f_{m+l}}{6}\right)-2 D\left(1-\frac{Q^{2} f_{m+l}}{2}\right)\left(k+c Q+\frac{w Q^{3} f_{m+l}}{6}\right)=0
\end{aligned}
$$




$$
\begin{aligned}
h Q^{2}- & \frac{h Q^{4} f_{m+l}}{3}+\frac{h Q^{6} f_{m+l}}{36}-\frac{D c Q^{3} f_{m+l}}{3}+\frac{D w Q^{3} f_{m+l}}{3}-\frac{D Q^{5} w f_{m+l}}{6}-2 D k-\frac{D w Q^{3} f_{m+l}}{3}+D Q^{2} k f_{m+l} \\
& +D c Q^{3} f_{m+l}+\frac{D Q^{5} w f_{m+l}}{3}=0
\end{aligned}
$$

$$
\begin{gathered}
\frac{h f_{m+l} Q^{6}}{36}+\left(\frac{D w f_{m+l}}{3}-\frac{D w f_{m+l}}{6}\right) Q^{5}-\frac{h f_{m+l} Q^{4}}{3}+\left(-\frac{D c f_{m+l}}{3}+D w f_{m+l}-\frac{D w f_{m+l}}{3}+D c f_{m+l}\right) Q^{3} \\
+\left(h+D k f_{m+l}\right) Q^{2}-2 D k=0
\end{gathered}
$$

Equation (2) is used to obtain the value of the order quantity.

Next, we present Izevbizua and Omosigho (2016) model.

\section{Model Assumptions and Description.}

1. Ordering of new products is based on the remaining useful lifetime of the items on hand. We place order for new items when the useful lifetime remaining on the items on hand is one period.

2. Only one order is placed at a time. There are no outstanding orders.

3. The new order arrives instantly, whenever orders are placed.

4. Order received is used to satisfy demand in period $1,2, \ldots m$.

5. The fixed lifetime of the product is a positive integer $m$

6. A complete cycle consists of two consecutive order receive. One at the beginning of the cycle and the other at the end of the cycle. The time in between is the cycle length.

7. A shortage occurs whenever the on-hand inventory is not able to satisfy all the demand in a period. Only a part of the demand is satisfied while the other part is lost. That is lost sale is assumed and a shortage cost is charged against the inventory manager.

8. Item(s) not used to meet demand by the end of the period $\boldsymbol{m}$, outdates and are discarded. An outdated cost is charged against the inventory manager.

9. The issuing policy is FIFO, that is the oldest units must be used to meet demand before the new ones are used.

10. All units of the new order are of the same age and arrive into the inventory at age zero.

Demand in each period are not known but assumed to be independent and identically distributed random variables $d_{1}, d_{2}, \ldots$ with known distribution $f$. The demand $t=\sum_{i=1}^{m} d_{i}$ has density $f^{*}$ which is the $n$-fold convolution of $f$ with itself. Table 1 gives a description of the useful lifetime based model for a product whose lifetime is $m_{\text {periods }}$

Table 1: Orders and Age distribution for a product with $\boldsymbol{m}$ lifetime

\begin{tabular}{|c|c|c|c|c|c|c|c|c|}
\hline Order & \multicolumn{1}{c|}{$T_{1}$} & $T_{2}$ & $T_{3}$ &. & $T_{m-1}$ & $T_{m}$ & $T_{m+1}$ & $T_{m+2}$ \\
\hline 1 & $\begin{array}{l}\left(y-d_{1}\right)^{+} \\
\text {age 1 }\end{array}$ & $\begin{array}{l}\left(y-\sum_{i=1}^{2} d_{i}\right)^{+} \\
\text {age2 }\end{array}$ & $\begin{array}{l}\left(y-\sum_{i=1}^{3} d_{i}\right)^{+} \\
\text {age 3 }\end{array}$ & $\ldots$ & $\begin{array}{c}\left(y-\sum_{i=1}^{m-1} d_{i}\right)^{+} \\
\text {age } m-1\end{array}$ & $\begin{array}{c}x-d_{m} \\
\text { age } m\end{array}$ & & \\
\hline 2 & & & & $\begin{array}{l}\left(y-d_{1}\right)^{+} \\
\text {age 1 }\end{array}$ & age 2 & & \\
\hline 3 & & & & & & & & $\begin{array}{c}\left.y-\sum_{i=1}^{2} d_{i}\right)^{+} \\
\text {age 0 }\end{array}$ \\
\hline
\end{tabular}

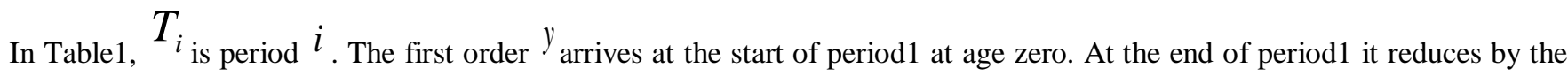
demand in period1 and becomes $\left(y-d_{1}\right)^{+}$, age1. The number of items brought into period2 is $\left(y-d_{1}\right)^{+}$and this reduces (by 
demand) in period2 and becomes $\left(y-d_{1}-d_{2}\right)^{+}$,age2. This continues until the reordering period $m-1$ when the first order \[ x=y-\sum_{i=1}^{m-1} d_{i} \text { and the second order arrives. Finally, at the } \]
reduces to
meet demand outdated leaving only items from the second order.
The shortage, outdate, and holding costs are as shown in equations (3),
Expected (unsatisfied demand) $=\int_{x+y}^{\infty}(t-(x+y)) f^{*}(t) d t$

Expected (outdate) $=\int_{0}^{x}(x-t) f(t) d t$

Holding $\cos t=h \int_{0}^{x+y}(x+y-t) f^{*}(t) d t$

There is a fixed ordering cost $k$ per unit ordered so that our ordering cost is $k y$.

Therefore, our total cost function is

$C(x, y)=\min _{y \geq 0}\left\{k y+h \int_{0}^{x+y}(x+y-t) f^{*}(t) d t+v \int_{x+y}^{\infty}(t-(x+y)) f^{*}(t) d t+\theta \int_{0}^{y}(y-t) f^{*}(t) d t\right\}$

where

$f^{*}(t)=$ distribution of total demand in periods $1,2, \ldots$. . that is

$f^{*}(t)$ is distribution of $t=\sum_{i=1}^{m} d_{i}$

$m=$ lifetime of the product. $m$ is a positive integer

$d_{i}=$ demand in period $i$

$t=\sum_{i=1}^{m} d_{i}$, total demand with distribution $f^{*}(t)$

$x$ represent the quantity of products with one useful period remaining in them. That is

$$
x=y-\sum_{i=1}^{m-1} d_{i}
$$

$y=$ new products ordered/entering into inventory with age zero

$\theta=$ outdate cost per unit

$v=$ shortage cost per unit

$h=$ holding cost per unit

$k$ = fixed ordering cost per unit.

To find the optimal order quantity, we minimize (6) with respect to the order quantity.

$\frac{\partial C}{\partial y}=k+h\left\{\frac{x^{2} f^{*}}{2}+x y f^{*}+\frac{y^{2} f^{*}}{2}\right\}-v\left\{1-\frac{x^{2} f^{*}}{2}-x y f^{*}-\frac{y^{2} f^{*}}{2}\right\}+\frac{\theta y^{2} f^{*}}{2}$

where $f^{*}(t)=\lambda e^{-\lambda t}$

$\left(\frac{h f^{*}}{2}+\frac{v f^{*}}{2}+\frac{\theta f^{*}}{2}\right) y^{2}+\left(h x f^{*}+v x f^{*}\right) y+k+\frac{h x^{2} f^{*}}{2}-v+\frac{v x^{2} f^{*}}{2}=0$

$\left(\frac{h}{2}+\frac{v}{2}+\frac{\theta}{2}\right) f^{*} y^{2}+(h x+v x) f^{*} y+k+\frac{h x^{2} f^{*}}{2}-v+\frac{v x^{2} f^{*}}{2}=0$

Equation (7) is used for obtaining the order quantity for our model. Next, we compare the sensitivity of the two models to changes in the ordering cost per unit, holding cost per unit and outdate price per unit using equations (2) and (7). Computer programmes were written in MATHEMATICA 8 for equations (2) and (7) to generate the values of the order quantities for Chiu and our model. 
Citation: Izevbizua O, et al., Comparing the Sensitivity of Quantity Based and Useful Lifetime Based Fixed Lifetime Inventory Models to Changes in Costs. Australian Journal of Basic and Applied Sciences, 13(8): 1-7. DOI: 10.22587/ajbas.2019.13.8.1

\section{Numerical Examples}

Example 1: In this example we vary the ordering cost per unit to see how the two models react to changes in the ordering cost.

Table 2: Comparing quantity order varying the ordering cost per unit.

\begin{tabular}{|c|c|c|c|c|c|c|}
\hline $\mathrm{s} / \mathrm{n}$ & $k$ or $c$ & $h$ & $\theta$ or $w$ & $\lambda$ & $Q$ & $y$ \\
\hline 1 & 2 & 1 & 1 & 10 & 54.46 & 64.69 \\
\hline 2 & 20 & & & & 54.33 & 56.74 \\
\hline 3 & 25 & & & & 54.30 & 54.33 \\
\hline 4 & 30 & & & & 54.26 & 51.79 \\
\hline 5 & 35 & & & & 54.23 & 49.14 \\
\hline 6 & 40 & & & & 54.20 & 46.33 \\
\hline 7 & 45 & & & & 54.16 & 43.34 \\
\hline 8 & 48 & & & & 54.14 & 41.44 \\
\hline 9 & 50 & & & & 54.13 & 40.12 \\
\hline 10 & 60 & & & & 54.06 & 32.75 \\
\hline
\end{tabular}

From Table 2, we observed that the higher the ordering cost per unit, the lower the quantity ordered by the two models. However, the useful lifetime-based model is more sensitive to changes in the ordering cost as it ordered fewer items than the quantity based model as the ordering cost increases. Since an increase in the ordering cost per unit imply an increase in the selling price per unit, which in turn imply a decrease in demand, ordering almost the same quantity even at higher cost can lead to increase in the number of outdates. Minimizing outdates is the main goal of research in the fixed lifetime inventory system.

Example 2: In this example we vary the holding cost per unit to see how the two models react to changes in the holding cost.

Table 3: Comparing quantity order varying the holding cost per unit.

\begin{tabular}{|c|c|c|c|c|c|c|}
\hline $\mathrm{s} / \mathrm{n}$ & k or $c$ & $h$ & $\theta$ or $w$ & $\lambda$ & $Q$ & $y$ \\
\hline 1 & 60 & 1 & 1 & 10 & 54.06 & 32.75 \\
\hline 2 & & 5 & & & 52.16 & 31.98 \\
\hline 3 & & 8 & & & 51.06 & 31.44 \\
\hline 4 & 10 & & & 50.43 & 31.09 \\
\hline 5 & 13 & & & 49.59 & 30.59 \\
\hline 6 & 17 & & & 48.63 & 29.96 \\
\hline 7 & & 20 & & & 48.00 & 29.52 \\
\hline 8 & & 25 & & & 47.09 & 28.81 \\
\hline 9 & & 28 & & & 46.61 & 28.41 \\
\hline 10 & & 30 & & & 46.31 & 28.15 \\
\hline
\end{tabular}

From Table 3, we observed that the higher the holding cost the lower the quantity ordered by the two models. Again, the useful lifetime based model order fewer items when compared with the quantity based model as the holding cost increases, thereby reducing the holding cost for the model.

Example 3: In this example we vary the outdate cost per unit to see how the two models react to changes in the outdate cost.

Table 4: Comparing quantity order varying the outdate cost per unit

\begin{tabular}{|c|c|c|c|c|c|c|}
\hline $\mathrm{s} / \mathrm{n}$ & $k$ or $c$ & $h$ & $\theta$ or $w$ & $\lambda$ & $Q$ & $y$ \\
\hline 1 & 60 & 1 & 1 & 10 & 54.06 & 32.75 \\
\hline 2 & & & 5 & & 44.60 & 31.99 \\
\hline 3 & & & 7 & & 42.44 & 31.62 \\
\hline 4 & & & 10 & & 40.16 & 31.09 \\
\hline 5 & & & 15 & & 37.60 & 30.28 \\
\hline 6 & & & 18 & & 36.48 & 29.81 \\
\hline 7 & & & 20 & & 35.83 & 29.51 \\
\hline 8 & & & 25 & & 34.50 & 28.81 \\
\hline 9 & & & 28 & & 33.42 & 28.15 \\
\hline 10 & & & 30 & & 32.53 & 27.54 \\
\hline
\end{tabular}

From Table 4, we observed that the higher the outdate cost per outdate, the lower the quantity ordered by the two models. However, for each outdate cost the useful lifetime-based model ordered fewer goods than the quantity based model. Finally, we compare the total cost of ordering for the two models over the planning horizon, using example 1. 
Citation: Izevbizua O, et al., Comparing the Sensitivity of Quantity Based and Useful Lifetime Based Fixed Lifetime Inventory Models to Changes in Costs. Australian Journal of Basic and Applied Sciences, 13(8): 1-7. DOI: 10.22587/ajbas.2019.13.8.1

Example 4: In this example we compare the total cost of ordering for the two models using the ordered quantities in example 1.

Table 5: Comparing ordering cost for the two models over the planning horizon.

\begin{tabular}{|c|c|c|c|c|c|c|}
\hline $\mathrm{s} / \mathrm{n}$ & $k$ or $c$ & $Q$ & $y$ & $\lambda$ & $c Q$ & $k y$ \\
\hline 1 & 2 & 54.46 & 64.69 & 10 & 108.92 & 129.38 \\
\hline 2 & 20 & 54.33 & 56.74 & & 1086.60 & 1134.80 \\
\hline 3 & 25 & 54.30 & 54.33 & & 1357.50 & 1358.25 \\
\hline 4 & 30 & 54.26 & 51.79 & & 1627.80 & 1553.70 \\
\hline 5 & 35 & 54.23 & 49.14 & & 1898.05 & 1719.90 \\
\hline 6 & 40 & 54.20 & 46.33 & & 2167.60 & 1853.20 \\
\hline 7 & 45 & 54.16 & 43.34 & & 2437.20 & 1950.30 \\
\hline 8 & 48 & 54.14 & 41.44 & & 2598.72 & 1989.12 \\
\hline 9 & 50 & 54.13 & 40.12 & & 2706.50 & 2006 \\
\hline 10 & 60 & 54.06 & 32.75 & & 3243.60 & 1965 \\
\hline Total & & & & & 19232.49 & 15659.65 \\
\hline
\end{tabular}

From Table 5, the total ordering cost for the useful lifetime based model after ten orders is 15659.65 while the total ordering cost for the quantity based model is 19232.49 . The ordering cost is higher for the quantity-based model because it is less sensitive to changes in the ordering price per unit. Instead of reducing the quantity ordered as ordering price increases, the model continue to order almost the same quantity. If demand fall as prices increase, then the number of outdates will rise.

\section{Discussion. \\ Outdate code}

During this research, the following were our observations.

Firstly, many inventory managers are involved in the repackaging of outdated products in order to maximize profit. Repackaging is the act of exchanging the original pack of a product with the right expiration date, with a new pack carrying a false or wrong expiration date. This is very common with inventory managers in the food and pharmaceuticals sectors.

In response to this, we recommend that the expiration date be written on the product and not on the pack.

Secondly, some products (especially pharmaceuticals) carry verification code on them. The code is sent to the producing or manufacturing company and an instant message sent back saying the product is authentic and safe for consumption. It, however, does not tell you whether the product has expired or when it will expire. We recommend that an expiration code be added to the verification code. The expiration code can be sent via sms to the producing company and an instant message sent back to the consumer with either one of the following messages (i) expired on the $2^{\text {nd }}$ April 2015, for an expired product or (ii) will expire on $2^{\text {nd }}$ April 2019, for a product that has not expired. The expiration code should be on the product and not on the pack. The verification code and the expiration code can be combined into one code called the Verification and Expiration Code or simply (VEC). The introduction of the expiration code and the verification code (already being practice) will make the products much safer for Nigerians. Together, the codes will tell you that the product is authentic and when it will expire.

\section{Conclusion}

Over the years the decision to reorder new items have been based on the quantity of items on hand (quantity-based models), we introduced a new ordering policy where the decision to reorder is based on the number of useful lifetime remaining on the items on hand (useful lifetime based model). Using Chiu's model as an example, we have compared the sensitivity of quantitybased model to the useful lifetime based model. Numerical examples show that the useful lifetime-based model is more sensitive to changes in costs and order fewer items at higher cost. Also, over a given planning horizon the total ordering cost for the useful lifetime based model is lower when compared to the quantity based model. Finally, we introduce the outdate code to the fixed lifetime inventory system, which will help prevent the consumption of outdated products.

\section{Reference}

Bookbinder .J .H and Cakanyildirim. M (1999); Random lead times and expected orders in inventory systems. European journal of operational research . vol 155, pp300-313.

Chiu, H.N. (1994); An Approximation to the Continuous review Inventory Model with Perishable Items and Lead Times. European Journal to Operational Research. Vol. 87. pp. 93-108.

Hariga, M. (2010); A single-item continuous review inventory problem with space restriction. International journal of production economics. Vol 128, pp 153-158.

Hollier et al (1995); Continuous review policies for inventory systems incorporating cutoff transaction size. International journal of production res. vol 33 (10), pp 2855-2865.

Liu, L. and Lian, Z. (1999); (s,S) Continuous Review Models for Products with Fixed Lifetime. Operations Research, Vol. 47(1). pp. 150-158.

Mohammad, H.A and Manuel, D.R (2007); An efficient heuristic optimization algorithm for a two -echelon inventory system. International journal of production economics vol 109,pp195-213.

Nahmias, S (1978); The fixed-charge perishable inventory problem. Journal of Operations Research. Vol 26, (3) pp464-481.

Nahmias, S. and Pierskalla, W. P. (1973); Optimal Ordering Policies for a Product that Perishes in two Periods Subject to Stochastic Demand. Naval Research Logistics Quarterly. Vol. 20(2). pp. 207-229. 
Olsson, F. and Tydesjo .P (2010): Inventory Problems with Perishable items: Fixed Lifetimes and Backlogging. European Journal of Operational Research. Vol. 202. pp .131-137.

Perry, D. and Posner, M.J.M. (1998); An (-1, S) Inventory System with Fixed Shelf life and Constant lead times. Operations Research Vol. 46(3), pp.65-71.

Schmidt, C. P. and Nahmias, S. (1985); (S-1, S) Policies for Perishable Inventory. Management Science. Vol. 31(6). pp. 719-728.

Shen, Z, Dessouky, M and Ordonez, F. (2012); Perishable inventory management system with a minimum volume constraint. Journal of the Operational Research Society. Vol 62, pp 2063-2082.

Silver, E.A, Bischak, D.P and Kok, T (2012); Determining the reorder point and the order-up-to level to satisfy two constraints in a periodic review system under negative binomial demand.

Siriruk, P (2012); The optimal ordering policy for a perishable inventory system. Proceedings of the world congress on engineering and computer science. Vol II.

Izevbizua, O and Omosigho, S.E (2016); The management of inventory system of products with fixed lifetime. unpublished Ph.D thesis submitted to postgraduate school University of Benin, Benin City 